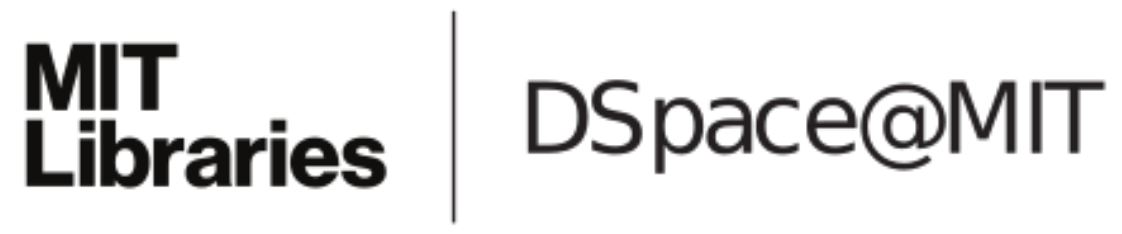

\author{
MIT Open Access Articles
}

The effect of amplitude (power) variations on beam combining efficiency for phased arrays

The MIT Faculty has made this article openly available. Please share how this access benefits you. Your story matters.

Citation: Fan, T.Y. “The Effect of Amplitude (Power) Variations on Beam Combining Efficiency for Phased Arrays." Selected Topics in Quantum Electronics, IEEE Journal of 15.2 (2009): 291-293. (C) 2009 Institute of Electrical and Electronics Engineers

As Published: http://dx.doi.org/10.1109/JSTQE.2008.2010232

Publisher: Institute of Electrical and Electronics Engineers

Persistent URL: http://hdl.handle.net/1721.1/52452

Version: Final published version: final published article, as it appeared in a journal, conference proceedings, or other formally published context

Terms of Use: Article is made available in accordance with the publisher's policy and may be subject to US copyright law. Please refer to the publisher's site for terms of use. 


\title{
The Effect of Amplitude (Power) Variations on Beam Combining Efficiency for Phased Arrays
}

\author{
Tso Yee Fan, Senior Member, IEEE
}

\begin{abstract}
In coherent beam combining (phased arrays), control of the relative amplitudes (powers) of array elements is needed to maximize the beam combining efficiency. Here, simple expressions are derived for the efficiency in approaches that use interferometric combiners such as diffractive optical elements or fiber couplers. The beam combining efficiency is relatively insensitive to small power errors. In the case of dropped elements, the efficiency is equal to the fraction of operating elements. The beam combining efficiency is equivalent to the Strehl ratio for a tiled aperture.
\end{abstract}

Index Terms-Laser arrays, optical arrays, phased arrays.

\section{INTRODUCTION}

C OHERENT beam combining (phased arrays) is becoming increasingly of interest as an approach for increasing the power and brightness from arrays of lasers [1]. While it is widely recognized that phase control is important for efficient combining, amplitude control is also important. The effect on efficiency of phase-control errors has been previously analyzed [2], [3]; here, an analysis of the effect of amplitude (power) control errors is presented, with the result that amplitude variations among the elements are not strong drivers of combining inefficiency. A simple expression is derived for the efficiency, which gives physical insight, similar to simple expressions that are often used to describe the effect of phase errors. In the limit that only some fraction of the laser array is operational, the beam combining efficiency (assuming the phase errors are 0) is simply equal to the fraction of the array that is operational. Although amplitude-control errors are not strong drivers of beam combining efficiency, this is a less favorable situation than wavelength beam combining, in which amplitude control has no effect on beam combining efficiency [1].

Coherent beam combining approaches can be generally classed between tiled-aperture and filled-aperture implementations. In the former, the array element outputs are placed spatially adjacent to each other and interfere with each other in the far field. In the latter, the outputs are overlapped at an interferometric beam combiner (such as a holographic beam splitter or an $N \times N$ fiber coupler). There is an equivalence between these two approaches, with the difference being whether the interference occurs in the near field or far field. The analysis presented

Manuscript received September 30, 2008; revised November 2, 2008 and November 12, 2008. First published February 10, 2009; current version published April 8, 2009. This work was supported by the Defense Advanced Projects Research Agency under Air Force Contract FA8721-05-C-0002 and by the Defense Advanced Research Projects Agency (DARPA) Coherently Combined High-Power Single-Mode Emitters (COCHISE) Program. Opinions, interpretations, conclusions, and recommendations are those of the authors and are not necessarily endorsed by the United States Government.

The author is with Lincoln Laboratory, Massachusetts Institute of Technology, Lexington, MA 02420 USA (e-mail: fan@11.mit.edu).

Digital Object Identifier 10.1109/JSTQE.2008.2010232
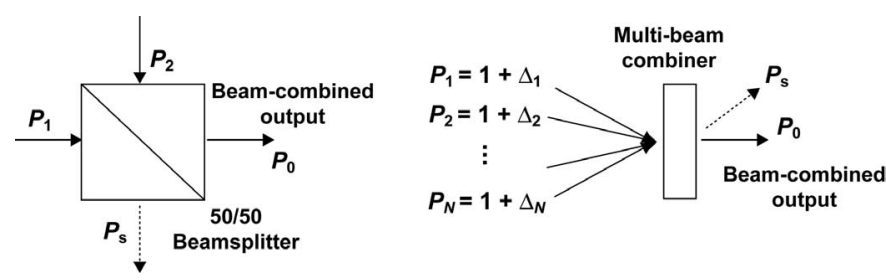

Fig. 1. Interferometric combining of laser beams. $P_{0}$ is the power in the combined beam and $P_{\mathrm{s}}$ is the power scattered in other directions. (Left) Combining two beams at a 50/50 beam splitter. (Right) Combining of multiple beams.

here is based on filled-aperture implementations, but the beam combining efficiency is entirely equivalent to the Strehl ratio for a tiled-aperture implementation.

\section{Two-ELEMENT BeAm Combining EFFICIENCY}

Consider the simplest case of beam combining pertaining to two elements at a 50/50 beam splitter, as shown in Fig. 1. Two beams are incident with powers $P_{1}$ and $P_{2}$ with the beamcombined output having power $P_{0}$. The power out of the undesired port is $P_{\mathrm{s}}$. It is assumed that the incident beams are coherent with respect to each other and that they are mode-matched. The output power is [2]

$$
P_{0}=\frac{1}{2}\left|\sqrt{P_{1}} \exp (j \phi)+\sqrt{P_{2}} \exp (-j \phi)\right|^{2} .
$$

Here $j=(-1)^{1 / 2}$ and $\phi$ is the relative phase. Here, $\phi$ is defined such that $\phi=0$ results in constructive interference at the output. Consequently, the beam combining efficiency $\eta$, which is the ratio of $P_{0}$ to the sum of the input powers, is given by

$$
\eta=\frac{P_{0}}{P_{1}+P_{2}}=\frac{1}{2} \frac{\left|\sqrt{P_{1}} \exp (j \phi)+\sqrt{P_{2}} \exp (-j \phi)\right|^{2}}{P_{1}+P_{2}} .
$$

The total input power can be normalized to $P_{1}+P_{2}=2$ where $P_{1}=1+\Delta$ and $P_{2}=1-\Delta$, with $\Delta$ being the difference in power from the average. For equal input powers, $\Delta=0$, and for one of the inputs being $0, \Delta=1$. For $\phi=0$, (2) can be rewritten as

$$
\eta(\phi=0)=\eta_{a}=\frac{1}{4}(\sqrt{1+\Delta}+\sqrt{1-\Delta})^{2} .
$$

In the limit of small $\Delta$, (3) can be series-expanded, and by retaining up through square terms in $\Delta, \eta_{a}$ can be approximated as

$$
\eta_{a} \approx 1-\frac{\Delta^{2}}{4} .
$$

Fig. 2 shows the efficiency as a function of $\Delta$ for both the exact and approximate solutions in (3) and (4), respectively. The 


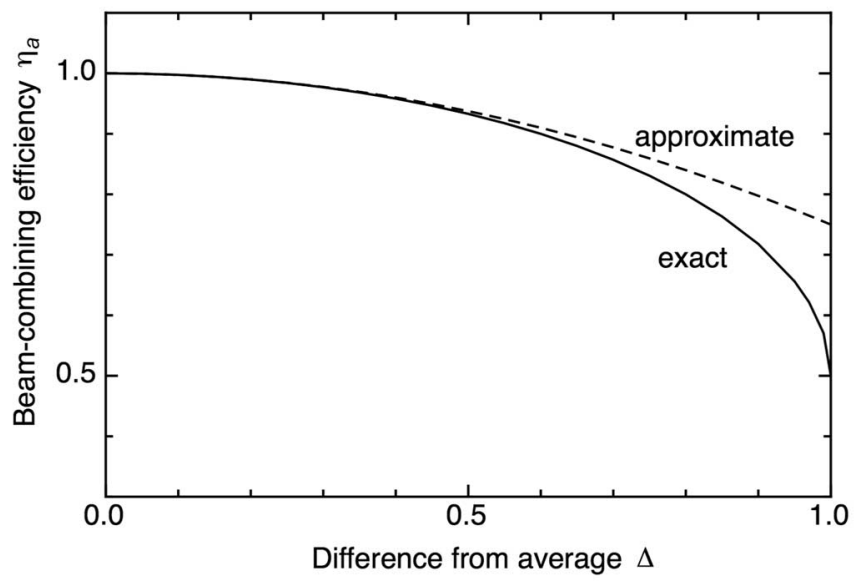

Fig. 2. Two-element beam combining efficiency as a function of $\Delta$ for $\phi=0$. The approximation of (4) is the dashed line, and the exact solution of (3) is the solid line.

approximation is very good up to $\Delta$ of around 0.6 . For $\Delta=0.6$ (representing a ratio of $P_{1} / P_{2}$ of 4 ), the beam combining efficiency is still 0.9 showing that relatively large power differences can be tolerated while still maintaining good beam combining efficiency.

\section{Generalization to $N$ Elements for SMall Power VARIATIONS AMONG ELEMENTS}

The results of (2)-(4) can be generalized to $N$ elements by considering the optical interferometric beam combiner as a multiport system using the scattering matrix formalism [4], [5] be it a holographic optical element [6], [7], a tree of 50/50 beam splitters [8], [9], or an $N \times N$ fiber coupler [10]. Here, we consider the specific case of a beam combiner in which equal powers are required of the inputs in order to get unity beam combining efficiency, and assume that the elements are mode-matched and the inputs are coherent with each other. This is idealized in Fig. 1.

The beam combining efficiency for a multiport system has been previously derived [6]; that work included the general case of a beam combiner that requires unequal powers to achieve unity beam combining efficiency. Here, the assumption of equal powers is made in order to derive a simple, intuitive expression for beam combining efficiency. In generalizing to $N$ elements, (2) becomes

$$
\eta=\frac{1}{N} \frac{\left|\sum_{m=1}^{N} \sqrt{P_{m}} \exp \left(j \phi_{m}\right)\right|^{2}}{\sum_{m=1}^{N} P_{m}}
$$

where $P_{m}$ and $\phi_{m}$ are the power and phase in the $m$ th beam, respectively ( $m=1$ to $N)$. By definition, it has been chosen that $\phi_{m}=0$ results in constructive interference to the output beam. This choice can be made because the reference plane for defining the scattering matrix coefficients can be chosen arbitrarily [5]. This choice does, however, impose conditions on the other components of the scattering matrix so that it is unitary [5]. For $\phi_{m}=0$

$$
\eta_{a}=\left[\frac{1}{N} \sum_{m=1}^{N} \sqrt{1+\Delta_{m}}\right]^{2}
$$

where $\Delta_{m}$ is the difference in power from the average for the $m$ th element. One important difference between the twoelement case and the $N$-element case is the range of $\Delta$. In general, $-1 \leq \Delta_{m} \leq N-1$. In the limit of small $\left|\Delta_{m}\right|, \eta_{a}$ can be approximated as

$$
\eta_{a} \approx 1-\frac{1}{4 N} \sum_{m=1}^{N} \Delta_{m}^{2} .
$$

In deriving this approximation from (6), $\left|\Delta_{m}\right|$ must be less than 1 and only the square terms of $\Delta_{m}$ are retained in the series expansion. Also, terms containing powers of $N$ greater than 2 (in the denominator) are ignored. In the limit of large $N$, in which the deviations can be expressed probabilistically, this becomes

$$
\eta_{a} \approx 1-\frac{\sigma_{\Delta}^{2}}{4}
$$

where $\sigma_{\Delta}$ is the standard deviation of $\Delta$, similar to (4). This is similar in form to the approximation of the beam combining efficiency in the case of small phase errors (but no amplitude errors) $\eta_{\phi}$, which is given by [2], [3]

$$
\eta_{\phi} \approx 1-\sigma_{\phi}^{2}
$$

where $\sigma_{\phi}$ is the standard deviation of the phase. It can be shown in the limit of small phase and amplitude errors that

$$
\eta \approx \eta_{a} \eta_{\phi}
$$

\section{EFFECT ON COMBINING EFFICIENCY FOR NONOPERATIONAL ELEMENTS}

This analysis can be used to determine the dependence of beam combining efficiency on the number of elements. Here, we want to understand what happens to the beam combining efficiency when only a fraction of the array is operational. The starting point for this analysis is (5). In the case where all elements have equal power and are all operational, $P_{m}=P_{1}$, and thus, $\eta=1$ for $\phi_{m}=0$. For only a fraction $F(F \leq 1)$ of the elements being operational, (5) becomes

$$
\eta_{a}=\frac{1}{N} \frac{\left[F N \sqrt{P_{1}}\right]^{2}}{F N P_{1}}=F .
$$

Hence, $\eta_{a}$ is equal to the fraction of operational elements, similar to the Strehl ratio being proportional to the fill factor for a tiled-aperture implementation [2]. Since the total power from the array is proportional to $F$, the power in the combined beam scales as $F^{2}$, which is the same result previously highlighted for the degradation of the performance as array elements fail. The beam combining efficiency can be made unity and the power in the combined beam degraded to only $F$ by changing the optical system such that the interferometric combiner is designed for FN elements [1]. 


\section{EQuivalence of BEAM COMBINING EFFICIENCY AND STREHL RATIO}

The beam combining efficiency for interferometric beam combiners is equivalent to the Strehl ratio for tiled apertures. Here, the strict definition of Strehl ratio is used, in which a uniformly illuminated aperture with uniform phase gives a Strehl ratio of 1 . The expression for beam combining efficiency [see (5)] is identical to the expression for the Strehl ratio for a tiled aperture [2]. Given the equivalence, some additional statements can be made regarding phase and amplitude errors for coherent combining. In general, the degradation in beam combining efficiency caused by the combination of amplitude errors and phase errors is not separable, except in the limit of small errors. In other words, for a given phase-error distribution among the elements, there may be a nonzero amplitude variation among the elements that maximizes efficiency. This can be seen, for example, in the simple case of combining two beams. For $\phi=\pi / 2$, the maximum beam combining efficiency arises from one of the inputs having a power of 0 (i.e., $\Delta=1$ ); equal power inputs ( $\Delta=0$ ) lead to a beam combining efficiency of 0 . However, it is also true that, for any amplitude distribution, the highest beam combining efficiency (Strehl ratio) always occurs for no phase error [11] (i.e., $\phi_{m}=0$ by the definition in this paper).

\section{SUMMARY}

The reduction in beam combining efficiency caused by amplitude-control errors is relatively small in coherent beam combining. For the case in which only a fraction of the elements operate, the beam combining efficiency is equal to that fraction, assuming no phase error. While the degradation of beam combining efficiency is small, it still is less favorable than wavelength beam combining in which the beam combining efficiency is independent of amplitude. The beam combining efficiency of an interferometric combiner is equivalent to the Strehl ratio in a tiled-aperture implementation.

\section{ACKNOWLEDGMENT}

The author thanks A. Sanchez for helpful suggestions.

\section{REFERENCES}

[1] T. Y. Fan, "Laser beam combining for high-power, high-radiance sources," IEEE J. Sel. Topics Quantum Electron., vol. 11, no. 3, pp. 567-577, May/Jun. 2005.

[2] J. R. Leger, "External methods of phase locking and coherent beam addition of diode lasers," in Surface Emitting Semiconductor Lasers and Arrays, G. A. Evans and J. M. Hammer, Eds. Boston, MA: Academic, 1993, pp. 397-433.

[3] C. D. Nabors, "Effect of phase errors on coherent emitter arrays," Appl. Opt., vol. 33, pp. 2284-2289, 1994.

[4] H. A. Haus, Waves and Fields in Optoelectronics. Englewood Cliffs, NJ: Prentice-Hall, 1984, ch. 3.

[5] A. E. Siegman, Lasers. Mill Valley, CA: University Science, 1986 , pp. $398-407$.

[6] J. R. Leger, G. J. Swanson, and W. B. Veldkamp, "Coherent laser addition using binary phase gratings," Appl. Opt., vol. 26, pp. 4391-4399, 1987.

[7] E. C. Cheung, J. G. Ho, G. D. Goodno, R. R. Rice, J. Rothenberg, P. Thielen, M. Weber, and M. Wickham, "Diffractive-optics-based beam combination of a phase-locked fiber laser array," Opt. Lett., vol. 33, pp. 354-356, 2008.

[8] A. A. Ishaaya, N. Davidson, L. Shimshi, and A. A. Friesem, "Intracavity coherent addition of Gaussian beam distributions using a planar interferometric coupler," Appl. Phys. Lett., vol. 85, pp. 2187-2189, 2004.

[9] H. Bruesselbach, D. C. Jones, M. S. Mangir, M. Minden, and J. L. Rogers, "Self-organized coherence in fiber laser arrays," Opt. Lett., vol. 30, pp. 1339-1341, 2005.

[10] A. Shirakawa, T. Saitou, T. Sekiguichi, and K. Ueda, "Coherent addition of fiber lasers by use of a fiber coupler," Opt. Exp., vol. 10, pp. 1167-1172, 2002.

[11] V. N. Mahajan, Optical Imaging and Aberrations, Part 2, Wave Diffraction Optics. Bellingham, WA: SPIE, 2001, pp. 53-56.

Tso Yee Fan (S'82-M'87-SM'96) received the B.S. degree in electrical engineering and materials science and engineering from Massachusetts Institute of Technology (MIT), Cambridge, and the M.S. and Ph.D. degrees in electrical engineering from Stanford University, Stanford, CA.

In 1987, he was a Staff Member at the MIT Lincoln Laboratory, Lexington, where he is currently the Associate Leader of the Laser Technology and Applications Group. He has contributed broadly in solid-state laser and nonlinear optics technology. He is widely recognized for his work in diode-pumped solid-state lasers, in the development of Yb:YAG lasers, in characterization of laser and nonlinear optical materials, and for advances in laser beam combining. From 1994 to 1999, he was the Topical Editor (Lasers) for Optics Letters. From 2005 to 2007, he was the Division Editor for the Lasers, Photonics, and Environmental Optics Division of Applied Optics.

Dr. Fan is a Fellow of the Optical Society of America (OSA). From 1994 to 1999, he was an Elected Member of the IEEE/Lasers and Electro-Optics Society (LEOS) Board of Governors. He was a member of program committees of numerous conferences, including the Chair of the LEOS Annual Meeting and the OSA Topical Meeting on Advanced Solid-State Lasers. 